\title{
ESCALAS DE LA PLANEACIÓN Y VULNERABILIDAD TERRITORIAL EN MÉXICO
}

\section{SCALES OF PLANNING AND TERRITORIAL VULNERABILITY IN MEXICO}

\author{
Blanca Rebeca Ramírez VelázQuez \\ Universidad Autónoma Metropolitana-Xochimilco, Departamento de Teoría y Análisis, Ciudad de México, México
}

\begin{abstract}
R E S U M E N : Desde finales del siglo XX se ha presenciado en México una planeación de corte neoliberal basada en el recorte de la intervención estatal en sectores importantes de la economía del país y el desmantelamiento de los instrumentos que se generaron anteriormente. En el presente ensayo se argumenta que las políticas neoliberales impuestas en diferentes escalas han favorecido un desarrollo frágil y dependiente y, por lo tanto, vulnerable (RAMÍREZ, 2010) a partir de la implantación de un nuevo modelo de desarrollo que guía el tránsito al futuro. En condiciones de vulnerabilidad, se pone en riesgo la posibilidad de reproducción de la base material de la producción del país y de la reproducción social de los grupos más desfavorecidos y de territorios completos que presentan lesiones físicas considerables en sus condiciones de evolución y transformación. Las políticas en sus diferentes formas y escalas contribuyen a la implementación de este tipo de desarrollo.
\end{abstract}

P A L A B R A S C L A V E : planeación neoliberal; desarrollo fragil y dependiente; vulnerabilidad; reproducción material; reproducción social; desarrollo vulnerable.

A B S T R A C T : Since the end of the $20^{\text {th }}$ century, Mexico has experienced considerable neoliberal planning, based on reducing state intervention within important sectors of the country's economy and dismantling previously constructed instruments. In the present article, it is argued that neoliberal policies imposed at different scales have favored a fragile, dependent and therefore vulnerable development (RAMIREZ, 2010) from the implantation of a new development model that guided towards the future. Under such conditions of vulnerability, there is therefore a possibility of the risk of reproducing the productive material base of the country and of the social reproduction of disadvantaged groups and territories that present considerable physical problems in their conditions to evolve and transform. Policies in their different forms and scales contribute to the implementation of this type of development.

K E Y W O R D S : neoliberal planning; fragile,cv dependent development; vulnerability; material reproduction; social reproduction; vulnerable development.

DOI: https://doi.org/10.22296/2317-1529.2017v19n1p179 
En la década de los años setenta del siglo XX, la dimensión de la pobreza fue un eje que se centró en estudios territoriales, asumiendo que ésta era la causa fundamental de las migraciones del campo a la ciudad en Latinoamérica (SINGER, 1975). Aunque fue una postura que perduró durante muchos años, para finales del siglo hubo autores que argumentaron el carácter de exclusión en el que se encontraba una gran masa de campesinos de algunos países, México incluido (RUBIO, 2003). Para 2010, los campesinos eran el 22.2\% de la población rural en el país (INSTITUTO NACIONAL DE ESTADÍSTICA, GEOGRAFÍA E INFORMÁTICA (INEGI), 2010), correspondiendo a América Latina el 20.4\% en 2015. Este porcentaje decreció de más de $4.7 \%$ en tal solo 15 años de acuerdo a las proyecciones de la Comisión Económica para América Latina y el Caribe (1999). Recientemente, las condiciones de desigualdad social que prevalecen en la sociedad y en los territorios mexicanos y latinoamericanos han generado visiones alternas que evidencian como los problemas sociales de la población se han incrementado no sólo en cantidad sino también en calidad. La aparición de la pobreza urbana, la desigualdad y la exclusión social en las ciudades, siendo este territorio el eje fundamental del desarrollo moderno del país, aparecen como procesos evidentes especialmente en las metrópolis del siglo XXI (RAMÍREZ; ZICARDI, 2008).

Esta perspectiva permite observar el paso cuantitativo de la desigualdad en sí misma a otro de tipo cualitativo que la evidencia no como parte integrante del proceso de desarrollo, sino como una dimensión de exclusión social que es el origen de la formación de un Estado de excepción permanente (DURAND PONTE, 2010), presente ya en la sociedad, particularmente en la mexicana.

En el presente ensayo se argumenta que las políticas neoliberales impuestas en el país, particularmente a partir de la última década del siglo XX, más que contribuir a consolidar un desarrollo estable e integral económica y socialmente en México, sólo han favorecido la generación de lo que consideraremos un desarrollo frágil y dependiente (RAMÍREZ, 2010) que favorece esta exclusión de territorios y de sectores de la población. El desmantelamiento de los instrumentos y políticas de intervención estatal han generado condiciones de vulnerabilidad, que ponen en riesgo la posibilidad de reproducción de la base material de la producción del país, la reproducción social de los grupos más desfavorecidos y de territorios completos que presentan ya lesiones físicas considerables en sus condiciones de evolución y transformación.

Para fundamentar esta aseveración, en primera instancia, se presenta el nuevo modelo de desarrollo neoliberal dependiente, que prioriza el sector terciario y servicios y los negocios que la producción generando sectores, territorios y grupos vulnerables; en segunda instancia se analiza el concepto de vulnerabilidad como condición de la fragilidad y el paso de la vulnerabilidad del desastre a la social y territorial; en tercera, se argumenta que para contender contra la vulnerabilidad territorial, las políticas territoriales de desarrollo deben de integrarse de manera diferencial, integral y distributiva. Se asume con estas reflexiones, que la vulnerabilidad puede ser aplicada a otros países de América Latina, dependiendo de las condiciones específicas del desarrollo que se han impuesto. 


\section{LAS ESCALAS Y FORMAS DEL MODELO DE DESARROLLO}

El modelo de desarrollo que se ha implementado en el país en los últimos años tiene diferentes formas de manifestarse en el territorio por sectores de la economía: primario, secundario y terciario, así como también se implementa con particularidades en las zonas rurales y las urbanas afectando las bases materiales de producción integral y poniendo en riesgo las bases materiales de la producción y de la reproducción social y territorial de territorios y regiones específicos, o bien de ciertos grupos sociales. Como base fundamental, se cambió de una estrategia en donde la modernización y la transformación nacional, basada en el crecimiento interno del país, por un nuevo paradigma de globalización y apertura hacia el exterior fomentando el producir para exportar acompańado del paradigma de la sustentabilidad como bases para proteger los recursos naturales y el cambio climático (RAMÍREZ, 2003).

El nuevo modelo de desarrollo se basa en una estrategia que deja de poner atención a la dimensión productiva a la de servicios, cambiando el eje central de su acción hacia la terciarización de la economía que se presenta de manera diferente en los sectores económicos y en los territorios (RAMÍREZ, 2006). Si se analiza por sectores, en la escala nacional se percibe un agotamiento fuerte de la agricultura mexicana desde la década de los años setenta, en donde el campo ha sufrido un deterioro constante en sus condiciones materiales de producción y reproducción. De haber sido exportador de productos agrícolas como el maíz, México se ha convertido en un importador de productos alimenticios básicos a expensas de la producción de forrajes para el ganado (BARKIN; BATT; DEWALT, 1991) y aún de alimentos básicos para la alimentación de la población. Varios autores, entre ellos Arroyo (1989), han documentado la pérdida de la autosuficiencia alimentaria y el auge de la ganadería desde finales del siglo XX, lo que ha generado una dependencia alimenticia que es ya estructural y, lo que es peor, se ha acrecentado a partir de la implantación del modelo neoliberal (CALVA, 2007).

$\mathrm{El}$ argumento es que el grado de complejidad alcanzado por el sector primario y el campo que lo sostiene ha generado condiciones de vulnerabilidad en diferentes formas y escalas. Si se analiza la superficie en la que se ha producido, se observa, que solamente el 10\% de la superficie del país esta cosechada dato que se mantiene estable y sin crecimiento en los últimos 5 años (SECRETARIA DE AGRICULTURA, GANADERÍA, DESARROLLO RURAL, PESCA Y ALIMENTACIÓN, 2010), que solamente existían 21,855,443 hectárea en producción, de las cuales nada más $18,706,240$ fueron cosechadas (INEGI, 2009). Esto equivale a decir que el $0.15 \%$ de la superficie total del país fue productiva. Las condiciones montañosas o desérticas con las que cuenta éste son parte del origen de esta situación, pero se ha incrementado también por la falta de incentivos: producción con apoyo crediticio o los precios competitivos, así como una estrategia que posicione al sector como fundamental para el desarrollo nacional y para salvaguardar la soberanía del país (RAMÍREZ, 2007).

La creciente importación de granos básicos, aunada a la de los destinados a la producción de insumos de ganado, es también un factor importante para la generación de vulnerabilidad a escala nacional. La falta de autosuficiencia alimentaria mostrada por la incapacidad de producir al menos lo que se consume internamente, es un factor 
de dependencia internacional que ha puesto al país en condiciones de fragilidad interna. Se argumenta, que en este nivel, la vulnerabilidad que se está presentando a escala nacional, donde la fragilidad se ha incrementado día a día por la falta de una estrategia económica y política orientadas a buscar la autosuficiencia alimentaria repercutiendo drásticamente en el incremento de los costos de los alimentos básicos y fomentando los agronegocios. La extensión de tierras improductivas es otro dato para calificar la vulnerabilidad, refiriendo a la cantidad de unidades de producción que no presentaron alguna actividad agropecuaria o forestal. En 2007, según el VIII Censo Agrícola, éstas ascendían a $26.6 \%$ del total de unidades productivas del país. De ellas, $17.5 \%$ eran de superficie agrícola y no fueron sembradas durante el ciclo primavera-verano. De las tierras que no fueron sembradas el $33.2 \%$ no se utilizó debido a la falta de dinero o apoyo; en $25.9 \%$ se "quería dejar descansar la tierra"; $10.1 \%$ no se usó por mal temporal y en $5.6 \%$ de las unidades de producción no hubo quien las sembrara; $4.08 \%$ no se utilizó por el suelo poco fértil y finalmente $0.9 \%$ por suelo erosionado (INEGI, 2009).

Datos proporcionados por la Secretaría de Medio Ambiente y Recursos Naturales (SEMARNAT) en 2005 demuestran, a partir de la evaluación de la pérdida de suelos, que los afectados por algún tipo de degradación alcanzan $47.7 \%$ de la superficie total del país, de ellos $5 \%$ presenta deterioro severo o extremo y $95 \%$ está ubicado en los márgenes ligero a moderado. Los principales procesos de degradación de los suelos en México son la química, la erosión hídrica y la eólica, responsables en conjunto de 87\% de la superficie afectada, con 34.9, 23 y 18.5 millones de hectáreas, respectivamente (ENCISO, 2008). Las causas de degradación son el cambio de uso del suelo hacia la agricultura y el sobrepastoreo (ambas con 17.5\%); la deforestación con $7.4 \%$ en tercer lugar, seguida de la urbanización $1.5 \%$. A estas actividades hay que agregar la mineria creciente que se ha expandido en todo el continente mediante la venta de los derechos de explotación a las compañías canadiénses y estadounidenses que sumaban 26,002 en 2013, habíendose incrementado en más de 5\% desde 2008 (MÉXICO, 2014), repercuten en la reducción de la cubierta vegetal, responsable de la conservación del suelo y se basan en el despojo de las tierras campesinas que tradicionalmente han ostentado los propietarios de ellas.

Si los problemas de la erosión y la degradación del suelo son graves, éstos se ven incrementados por las 120 millones de hectáreas que han sido afectadas por la desertificación y la pérdida de fertilidad que se presenta en $18 \%$ del territorio nacional; la erosión hídrica es de $12 \%$, la eólica de $1 \%$ y la salinización de las tierras de riego de 8\%, lo que origina 530 millones de toneladas de azolves al ańo (ENCISO, 2008).

La deforestación en México se calcula en aproximadamente 500 mil hectáreas y provoca la inutilización de 10 mil por salinización, esto reduce el rendimiento de las cosechas a menos de la tercera parte del potencial de la tierra o repercute en la disminución de la capacidad de carga en predios de pastoreo, a menos de la cuarta parte. Asimismo, la producción forestal disminuye a menos de un décimo el potencial técnico de los bosques y selvas del país. Las causas de 93\% de la degradación de la tierra fueron las malas técnicas de manejo, como es el sobrepastoreo, la falta de planeación en el aprovechamiento forestal, el uso inadecuado y excesivo de maquinaria, la aplicación de agroquímicos, la roturación inadecuada, el laboreo excesivo y la mala aplicación del riego, por lo que el problema sólo podrá resolverse con el cambio de utilización de las tierras. (ENCISO, 2008). 
A este panorama de disminución de la capacidad productiva del soporte tierra, hay que agregar la falta de una política de apoyo a los precios de producción agrícola, que asegure la posibilidad de contender por la ganancia necesaria para producir. Esto ha provocado el desvío de cultivos hacia otros más rentables como la floricultura, las drogas, que constituyen una alternativa para quienes pueden producirlos. De acuerdo a cálculos proporcionados por el Senado de la República, se estima que en 2009 la superficie de cultivo destinada a la producción de droga "aumentó 35\%, lo que equivale a cerca de 12 mil hectáreas", aumentando, además, no sólo el consumo internacional sino también el interno (REVISTAEMET, 2011).

La forma intensiva de producción que se introdujo en una parte del campo, aunado a otras urbanas no ha tomado en cuenta la necesidad de preservar los suelos y el agua, originando su degradación y contaminación. Según el INEGI (2000), la superficie de suelo alterado por causas de contaminación a principios del siglo XXI fue de 25,967 km2, en donde los productos derivados del petróleo y la gasolina son las principales causas con el $63 \%$, a la que se suma la provocada por amoniaco, hidrocarburos, metales y solventes .

Se presentan, además, condiciones de vulnerabilidad en la familia, sobre todo las campesinas, cada día en condiciones más difíciles para reproducirse pues lo que se extrae del campo, poco toma en cuenta las necesidades de la reproducción de la población. Con ello se percibe la falta de una política que apoye la producción agrícola campesina en particular lo que genera un sector excluido de la política de desarrollo nacional, así como a sus agentes sociales (RUBIO, 2003).

De acuerdo con la Encuesta Nacional de Ocupación y Empleo (ENOE), para finales del 2008, el campo mexicano contendía con 5,866,706 personas, de ellas $70 \%$ (4,157,354) vivía en localidades rurales, es decir, de menos de 2,500 habitantes, que presentan los mayores indicadores de marginación y pobreza alimentaria en el país. En esta fuente se observa que en el cuarto trimestre de 2008, 1,964,593 de los trabajadores ocupados en el sector agropecuario tuvieron ingresos de sólo un salario mínimo y 1,203,424, de uno a dos salarios mínimos. En suma, 7 de cada 10 trabajadores del sector primario percibieron ingresos que los sitúa en alguno de los grados de pobreza indicados por el Consejo Nacional de Evaluación de la Política de Desarrollo Social (CONEVAL) (MERCOSUR, 2011). El 27\% del total de personas dedicadas a la agricultura se ocupa en la de subsistencia, y 1 de cada 4 ocupados en este sector tenía 60 años, que equivale a 17.4\% del total de la población del sector (MERCOSUR, 2011).

En los últimos años se ha reactivado la minería a partir de concesionar la explotación a compañías extranjeras, fundamentalmente canadienses y estadounidenses. Al quedar estas minas en tierras campesinas, se origina un conflicto fuerte entre los propietarios de la tierra (ejidatarios o campesinos) y el Estado nacional quien ostenta la propiedad de las minas por constitución. Con estas concesiones los mineros se apropian del agua y de la tierra que las contiene, dejando sin actividad productiva a los campesinos y terratenientes. Estas dotaciones se han incrementado en $5.2 \%$ entre 2008 y 2013, incrementando la superficie concesionada en $18.2 \%$ en el mismo periodo y contribuyendo a la contaminación de suelo y de agua en las zonas en donde se ubican (MÉXICO, 2014).

En conclusión el soporte en el que se basa la actividad productiva primaria, tanto agrícola, forestal y minera se encuentra, por un lado, privatizado, desgastado 
y degradado, o por el otro abandonado y con poca productividad. El cambio de la producción agrícola por la pecuaria y la falta de incentivos económicos que promuevan la producción (CALDERÓN; RAMÍREZ, 2002) son algunas de las causas que han generado el abandono del campo y la desviación de la fuerza productiva hacia otras actividades, sobre todo terciarias. Este es el sector de la producción que menos empleo genera y contiende con los de menor calidad. Se podría afirmar que el "vaciamiento productivo" del que hablan Quintana, Egurrola y Hernández (2007, p. 267) no sólo se produce en el sector manufacturero sino también en el sector primario que tiene, desde hace 40 años la tendencia de desgaste en sus soportes materiales, principalmente suelo y agua, así como en la disminución en su producción. Con ello se amenaza la sustentabilidad territorial de algunos lugares y secotres del campo.

El modelo de desarrollo neoliberal contemporáneo presenta fragilidad también en el sector secundario y terciario ya que si la industrialización fue el paradigma en el que se basó el crecimiento económico y urbano del modelo de sustitución de importaciones, la desindustrialización relativa o el cambio de localización de la industria es el proceso que sustenta al modelo neoliberal globalizador actual, que los sustituye por una terciarización de la economía. Si el modelo anterior centró su actividad en las principales metrópolis del país: el Distrito Federal, Guadalajara, Monterrey y Puebla, el contemporáneo se amplia hacia sus áreas metropolitanas como fueron el Estado de México, Tlaquepaque, o Tlaxcala. Pero el crecimiento urbano ampliado, formando sistemas urbanos regionales ampliados, la especialización de las urbes y el incremento de la demanda de suelo urbano para la vivienda originaron que el proceso se trasladara a los bordes urbanos con el consabido cambio de uso del suelo y la expansión de urbes dispersas de gran tamaño.

La desindustrialización relativa iniciada desde la última década del siglo XXI, afectó en un principio principalmente a la ciudad de México, ya que algunos autores llamaban la atención sobre este proceso desde 1994 (HIERNAUX; TOMAS, 1994), pero fue hasta 2002 en que en la ciudad de México fue caracterizado como desindustrialización, terciarización, informatización y contracción del mercado interno (FIDEICOMISO DE ESTUDIOS ESTRATÉGICOS SOBRE LA CIUDAD, 2002), refiriéndose a la sustitución del terciario por el sector industrial y la contracción del mercado interno que acompañó este cambio en el país.

Para Quintana, Egurola y Hernández (2007) este proceso constituye un "vaciamiento productivo" de los territorios, que se presenta no sólo por la destrucción o cierre de núcleos productivos, sino también por el debilitamiento que éste presenta en diferentes partes del país de manera diferencial: Veracruz, la quinta zona más importante entre 1988 y 1998 hubo una pérdida de 97 núcleos productivos a nivel nacional, de los cuales el $23.7 \%$ se localiza en la Región Centro del país, incluyendo la zona metropolitana; la región centro Pacífico con $18.5 \%$, Noreste con $24.4 \%$, el Noroeste con $15.4 \%$ y el Golfo Caribe con $12.3 \%$; a la Sur Pacífico le corresponde el $1.5 \%$, ya que es la zona menos industrializada del país (QUINTANA; EGUROLA; HERNÁNDEZ, 2007, p. 282). Esta tendencia sin duda se ha incrementado por factores diversos que será necesario analizar en detalle en otro momento.

Este cambio es importante pues significa una forma específica de abandonar las actividades que producen valor (el primario y el secundario) para adoptar otras que solamente lo distribuyen, como son los servicios o actividades terciarias. En este cambio el incremento de la dependencia para obtener insumos y productos 
generados en los sectores productivos es fundamental para contender con una falta de independencia, dados los alcances que esta transformación tiene. Este proceso es positivo por diversas razones: por ejemplo, Harvey (1989) propone que para recomponer el proceso productivo es necesario primero desindustrializar y luego renovar o reutilizar. Méndez (apud MÁRQUEZ, 2005), por su parte plantea que es una manifestación de la crisis industrial que se presenta en la actualidad o bien es parte del cambio que hay entre el posfordismo y el neofordismo contemporáneos. Para Coriat apud Garca (2006), puede ser indicio solo de un proceso inferior y más lento de productividad que el industrial o bien la característica fundamental con la que contienden en la actualidad las metrópolis.

A pesar de todas estas aseveraciones, es indiscutible que cierto grado de vulnerabilidad se genera en estos territorios que dejan de ser productivos, dada la dependencia que se tiene de otros lugares para la obtención de sus requerimientos. La disminución de los salarios y la falta de prestaciones con que contienden los trabajadores en su relación con el sector servicios llama la atención de algunos investigadores como Fernández (2011) interesados en la dependencia de grupos de trabajadores asentados en las periferias urbanas del trabajo urbano, que genera menores niveles de ingreso y hasta desocupación y desempleo que otros que tienen mayores opciones de trabajo en el área rural o el comercio (FERNÁNDEZ, 2011).

En este giro, que está marcando sin duda la manera como se vislumbra el futuro de los territorios en el país, lo más preocupante es la importancia tan marcada que se le ha dado al turismo, en sus diferentes vertientes: de playa, patrimonialista de bienes históricos, o ecológico, rural o urbano en sus diversas modalidades. En cualquiera de ellos, destaca el recurso natural con el que cuentan; el del patrimonio histórico y cultural que los territorios tienen como base para generar una actividad económica en el lugar.

Organismos internacionales como International Council on Monuments and Sites (ICOMOS) y la formulación de la Carta de Venecia dan valor a estos elementos, ya que por ejemplo el patrimonio es considerado como "la riqueza y la diversidad" que presenta una ciudad o un lugar y que debe ser aprovechado en beneficio de sus habitantes; es considerado recurso no renovable, cuyo aprovechamiento puede ser la base de la sustentabilidad y de su conservación (DÍAZ; GONZÁLEZ, 2005). Esta consideración de igualdad entre la naturaleza y el patrimonio es la que está en la base del turismo, como una actividad que se está transformando, incluso para algunos países como España es en una de las bases fundamentales de su desarrollo.

En México, esta visión ha proliferado desde 1993, con el cambio de modelo económico a uno en donde el libre comercio y la apertura internacional son la base de su transitar junto con el turismo internacional, y fundamentalmente el de playa; de hecho, éste se constituyó en un pilar importante de las divisas que el país necesitaba para el pago de su deuda externa (RAMÍREZ; PRADILLA, 1993). La transformación de territorios para el desarrollo de esta actividad datan de la década de los setenta, cuando la apertura de la zona turística del Caribe mexicano, con Cancún como centro, derivó en la construcción de una de las zonas más prósperas del país, a partir del turismo de invierno para visitantes de países norteños.

Llama la manera como este cambio se da en las zonas urbanas ya que parecería que el vaciamiento productivo que se ha dado en los centros de la ciudad se ha acompańado por una importancia patrimonialista de recuperar los monumentos 
históricos para fomentar el turismo y en menor grado la vivienda de algunos sectores. A este proceso se le ha denominado gentrificación, copiando las categorías y procesos que se dan en los países norteños, sin que realmente se haya comprobado que existe, que es de la misma manera y que podemos llamarle así. Lo que si es evidente es la reutilización que hay de los centros copiando las formas de reproducción de ciudades como Nueva York, y otras de Europa que definen un modelo de desarrollo urbano basado en servicios altamente especializados y concentrados que impactan de manera importante la estructura económica del país y de las ciudades, especialmente la Ciudad de México.

Pero ¿qué importancia tiene esta especialización en la conformación de territorios vulnerables en el país? La fragilidad con la que las ciudades y regiones turísticas en las ciudades y en las regiones como el sureste mexicano responden a la disminución los turistas que visitan las zonas, disminuyendo salarios y horas de trabajo por ejemplo, hacen evidente el riesgo que corren los territorios que basan su desarrollo exclusivamente en la actividad terciaria (RAMÍREZ, 2010), en la falta de autosuficiencia alimenticia y manufacturera, que pueden en cualquier momento quedar en "vaciamiento productivo y laboral" aún por condiciones naturales al centrar su reproducción en una sola actividad de servicios como el turismo. En suma, un modelo de desarrollo dependiente, frágil y sin producción, es la base de lo que produce la vulnerabilidad territorial en los territorios. ¿¿Qué significa ser vulnerable?

\section{DE LA VULNERABILIDAD NATURAL A LA SOCIAL Y TERRITORIAL}

En la década de 1980 se dio gran importancia a la forma como los efectos de fuerzas naturales, sismos, ciclones, derrumbes, lluvias torrenciales, o inundaciones, que empezaron a irrumpir con mayor fuerza en el mundo y en el país. Algunos investigadores sociales replantearon la manera de orientar el conocimiento de su impacto muy influenciados por las consecuencias del sismo de 1985 en la Ciudad de México (PLIEGO, 1992). Esta manera, se percibió que los desastres cada día afectaban más a grupos sociales que se encontraban en condiciones de pobreza, que contendían con efectos desiguales de emergencia y de reconstrucción ante estos eventos naturales que provocaban diversos siniestros y afectaban a diversos territorios. Una de las conclusiones fundamentales a las que se llegó en este momento, fue que la pobreza y las condiciones de ubicación y de instalación de los soportes materiales que la reproducen (vivienda fundamentalmente) eran las causas que generaban las condiciones de vulnerabilidad social que colocaba en un primer plano el proceso de riesgo-desastre (MACÍAS, 1992) de la sociedad y no a la inversa.

Una definición más genérica de vulnerabilidad, permite dar cuenta que es una cualidad de objetos o personas que tienen como característica fundamental que pueden ser heridos/as o recibir lesiones físicas o morales con extrema facilidad (REAL ACADEMIA DE LA LENGUA, 2001, p. 2321). El Diccionario Oxford considera que vulnerable es no estar protegido contra algún ataque o contra alguna crítica (HORNBY, 1974, p. 961).

Si bien estas dos definiciones coinciden con la de vulnerabilidad socioeconómica 
de 1982, la diferencia fundamental es que esta última centraba su atención en que la fragilidad en que se quedaba la población, independientemente de que el estrago afectara directamente al soporte material de su reproducción (MACÍAS, 1992). Esto hacia que la vulnerabilidad se convirtiera en catástrofe, ya que la sociedad generaba una incapacidad para reajustar los efectos del siniestro, es decir, presentaba "inflexibilidad o incapacidad para enfrentar el cambio" (MACÍAS, 1992, p. 5). La vulnerabilidad social propicia inseguridad, debilidad y exposición desventajosa frente al peligro; éste, dependiendo de su interacción, determina un riesgo, que se convierte en una condición dinámica (MACÍAS, 1992) en la medida en que se incrementa constantemente en tiempo y espacio.

A la fecha, se perciben tres cambios importantes en relación a la manera como se percibía la vulnerabilidad hace 30 años. El primero, a pesar de que se sigue afirmando que la población cae en estados de vulnerabilidad, se ha ampliado y argumenta que ésta no es solamente propia de la población o del soporte material que favorece su reproducción (la vivienda, por ejemplo), sino que, la fragilidad o la lesión en las condiciones en que se reproduce el neoliberalismo contemporáneo ya ha generado afectaciones en soportes materiales productivos y se extiende a territorios o regiones completas. Esto se percibe en los tres sectores de la economía de diferentes maneras como se ejemplificó anteriormente.

El segundo cambio se centra en aceptar que la familia ya no es la unidad exclusiva de análisis de la vulnerabilidad (MACÍAS, 1992), sino que tiene diferentes escalas en su reproducción. En la propuesta que aquí se presenta, la fragilidad no desconoce los territorios rurales de los urbanos; por el contrario, ambos de diferente manera se ven involucrados en procesos de desgaste que ponen en riesgo su reproducción física, social y económica, lo que los sitúa en condición de vulnerabilidad. La escala de fragilidad en que se encuentran diferentes territorios es múltiple y dinámica, y ahora puede también estar centrada en territorios y no sólo en personas.

El tercer cambio plantea que la condición de vulnerabilidad actualmente considera también a los recursos no materiales y los derechos que cada ciudadano tiene para contender con su transformación y desarrollo integral como ser humano. Ésta se asocia a ciertas desventajas y riesgos que se han incrementado en el caso de las mujeres de grupos sociales con menor poder social, en cuanto al acceso a la propiedad, al mercado laboral y a los ingresos (acceso a los recursos), ya que para ellos persisten mayores responsabilidades en el hogar - cuidado y tareas cotidianas - que condicionan su acceso a la ciudad (CZYTAJLO, 2011). En este sentido, la vulnerabilidad no es solamente una condición externa de las personas, familias o territorios, sino también de la sociedad que se pone en fragilidad social cuando se integran a ésta grupos enteros afectados por el daño, o los territorios que se hacen vulnerables al no presentar actividad económica, "vaciamiento productivo", generado por el debilitamiento productivo (QUINTANA; EGURROLA; HERNÁNDEZ, 2007) o bien por la especialidad económica centrada en el turismo (RAMÍREZ, 2010).

Desde esta perspectiva, se asume que hay un cambio importante del concepto tradicional de vulnerabilidad por uno que supone el aumento de la fragilidad de las personas o las familias respecto de ellas y no solamente es la fragilidad causada por condiciones externas, sino también internas. Alude a la carencia de recursos para enfrentar los riesgos y evitar mayores pérdidas, a decir de Ramírez (2010, p. 130): "La vulnerabilidad significa no una mera carencia o necesidad. Es estar sin 
defensas, inseguro, expuesto a riesgos, al shock y la presión y logra captar algunos de los aspectos multidimensionales, dinámicos y estructurales de la pobreza, el desgaste de los recursos o la imposibilidad de contender con la contingencia”. Incluye también a los territorios que pierden su capacidad de respuesta hacia los embates que imponen condiciones externas a su propia reproducción.

Aplicando este punto de vista a la vulnerabilidad territorial, el aporte de Czytajlo (2011) lo amplia al aceptar que contiende en dos dimensiones: la condición y la posición. La primera refiere a una situación material de vida, es decir, a la fragilidad urbana y a la rural, a contender con tierras erosionadas o desgastadas para la producción, privaciones de servicios y bienes básicos, o la falta de acceso a la educación, al empleo y la capacitación, al desempleo o a la excesiva carga de trabajo y a la falta de disponibilidad de tiempo. La segunda dimensión se relaciona con la ubicación y reconocimiento social asignado a los territorios respecto a los sectores productivos y las condiciones sociales de sus habitantes (territorios indígenas y rancherosu otros); a la inclusión o exclusión de los espacios en las políticas púbicas y la toma de decisiones y participación política; en la igualdad o desigualdad de salarios por el mismo trabajo en los sectores productivos de diferentes territorios; a las oportunidades para transitar autónomamente con respeto a las decisiones que personal o colectivamente se tomen frente a los procesos, entre otros aspectos que se encuentren en los procesos.

Es evidente que la falta de una política que lleve a los territorios a plantear una producción que se adecúe a las condiciones que mejor los desarrollen, a contender con una política integral para encontrar autosuficiencia y libertad de acción, y la falta de una transformación autosuficiente e integral de sus habitantes nos lleva a plantear que hay una contrapolítica territorial que, con el neoliberalismo, no sólo carece de una estrategia clara para orientar el desarrollo de las regiones y los territorios, sino que revierte las condiciones del modelo de sustitución de importaciones que había logrado un crecimiento económico del país en la primera mitad del siglo XX.

¿Cómo resolver estas contradicciones que se presentan en el desarrollo que en las condiciones actuales están generando no sólo desigualdades, sino también dependencia, fragilidad y por lo tanto, vulnerabilidad social y territorial? Si bien el contender por la adopción de derechos es un factor individual indispensable, también es cierto que al contender por factores de índole social y territorial donde la intervención del Estado es un factor fundamental para resolverlas.

\section{PLANEACIÓN INTEGRAL, DISTRIBUTIVA Y DIFERENCIAL}

La manera como el Estado mexicano ha salido de la escena de la planeación ha dejado en manos del capitalismo neoliberal nacional o internacional el desarrollo económico del país. Esto ha agudizado la generación de contra-políticas territoriales, que han puesto en condiciones de vulnerabilidad a diversos sectores de la población y a los recursos naturales que difícilmente pueden entrar en el proceso productivo.

El sector servicios, y en particular el turismo, marca el desarrollo en el ámbito de la reproducción social de la población y no en el de la producción, que cuando no se ve acompañado por una visión integral que contienda con otras actividades productivas 
puede fácilmente entrar en crisis y generar riesgos, así como vulnerabilidad territorial. Es por ello que es preciso contender con una política que favorezca la diversidad de las actividades económicas, buscando una estrategia integral y distributiva de los recursos generados.

La vulnerabilidad y la fragilidad se agudizan cuando se generan condiciones de dependencia que contienden con el desarrollo de las regiones y las hacen obedecer a los dictados del capitalismo internacional para lograr su desarrollo. Estas condiciones se presentan en el tipo de turismo que se escogió para implementar el "milagro mexicano" del sureste del país en regiones de la Riviera Maya, por ejemplo (RAMÍREZ, 2010).

Evidentemente éste es un desarrollo muy limitado, pues tiene una visión única en donde hay sectores excluidos que no contienden con tendencia de crecimiento o transformación porque ya no son considerados como prioritarios o porque son vistos como obsoletos, como la actividad campesina, como resultado de la dependencia alimentaria a la que se ha sometido el país con las importaciones de los excedentes de maíz, trigo y otros productos, como el petróleo de los países desarrollados.

El Estado contiende con ellos a partir de otros programas que calificaremos de asistencialistas, que sólo palían la extrema polarización de la sociedad, aunque ésta, a su vez, busca otras estrategias para su propio desarrollo. Entre las primeras están las que proporcionan los programas como son los de oportunidades, procampo, procede el seguro popular y la agenda alimentaria, que tendrán que ser analizados en sus alcances y consecuencias en otro momento.

Con mayor razón consideramos que sigue vigente la necesidad de contender con una estrategia de política territorial nacional que integre todas las visiones y sectores para dar oportunidad a los agentes y a los territorios de contender con su transición y su cambio de futuro. Con lo anterior, será necesario que las diversidades sean integradas e integrales, en donde la especialización no sea exclusión de otras actividades, sectores o grupos sociales de la región y el factor de distribución sea uno de los principios que rigen dicha actividad implementada por un Estado que asuma su responsabilidad en cada una de las escalas en donde su ubica (RAMÍREZ, 2007).

Coincidimos con Harvey (2000, p. 229) cuando argumenta que hay una "ausencia de un obvio proyecto de cambio social" y de un mecanismo de planificación que lo esté buscando. Pero al mismo tiempo existe en su opinión una falta de articulación entre las reivindicaciones que se ubican en la escala de la globalización internacional con lo que llama utopismos de proceso que son los que vislumbran los cambios nacionales y las transformaciones económicas y sociales en la escala nacional, con los juegos espaciales que materializan los procesos de cambio y que permiten expresar, explorar y cambiar una gama amplia de relaciones sociales que transforman las formas espaciales concretas de territorios, sean urbanos, rurales en la escala de lo local (RAMÍREZ, 2003). Esa falta de integración entre las tres escalas de la planeación y las contradicciones que se generan en el proceso, es lo que produce la fragilidad y vulnerabilidad de los territorios que tanto afecta la posibilidad de un cambio justo y equitativo no sólo en el territorio mexicano, sino también en muchos territorios del mundo y en especial en los latinoamericanos.
Blanca Rebeca Ramírez Velázquez es licenciada en Geografía por la Universidad Nacional Autónoma de México (UNAM); posee Maestría en Planeación Regional Rural por la University of Aberdeen (Gran Bretaña); posee Doctorado en Urbanismo por la UNAM profesora-investigadora en el Departamento de Teoría y Análisis de la UNAM, unidad Xochimilco.

E-mail: blare19@me.com

Artículo recibido el 30 de septiembre de 2016 y aprobado para publicación el 2 de diciembre de 2016 . 


\section{REFERENCIAS}

ARROYO, G. (Coord.). La pérdida de la autosuficiencia alimentaria y el auge de la ganadería en México. México: Plaza u Valdés editores; UAM-Xochimilco, 1989.

BARKIN, D.; BATT, R.; DEWALT, B. Alimentos versus forrajes. La sustitución entre granos a escala mundial. México: Siglo XXI; UAM-Xochimilco, 1991.

CALDERÓN, G.; RAMÍREZ, B. R. De campesino jornalero a yuntero: neoliberalismo y "desarrollo" en el campo mexicano. In: SEGRELLES, J. A. (Coord.). Agricultura y espacio rural en Latinoamérica y España. Posibilidades y riesgos ante la mundialización de la economía. Madrid: Ministerio de Agricultura, Pesca y Alimentación, 2002. p. 265-322.

CALVA, J. L. Políticas de desarrollo agropecuario. In: (Coord.). Agenda para el desarrollo. México: UNAM; Miguel Ángel Porrua, 2007. p. 17-33.

COMISIÓN ECONÓMICA PARA AMÉRICA LATINA Y EL CARIBE. Boletín Demográfico. Santiago: CEPAL, 1999.

CZYTAJLO, N. El papel de las políticas habitacionales en la construcción de ciudades más democráticas. Discusiones desde la perspectiva de género. Ciudades, n. 89, p. 2-15, ene./ mar. 2011.

DIAZ, S.; GONZALEZ, A. De una expansión indiscriminada a una planeación que incluya y respete el patrimonio cultural y natural. In: SEMINARIO DE REPENSAR LA METRÓPOLIS, 1., 2006, Ciudad de México. Anales... Ciudad de México: Universidad Autónoma Metropolitana, oct. 2005.

DURAND PONTE, V. M. Desigualdad social y ciudadania precaria ¿Estado de excepción permanente? México: UNAM, Instituto de Investigaciones Sociales; Siglo XXI, 2010.

ENCISO, A. Soslaya el gobierno grave avance de la desertificación en México. La Jornada, 18 jun. 2008. Disponible en: <http://www.jornada.unam.mx/2008/06/18/index.php?section $=$ sociedad $\&$ article $=048 \mathrm{n} 1$ soc $>$. Acceso en: 15 diz. 2016.

FERNÁNDEZ, P. La relación campo-ciudad en las localidades del borde sur de la Zona Metropolitana del valle de México. Tesis (Doctorado en Ciencias Sociales) - Universidad Autónoma Metropolitana-Xochimilco, Ciudad de México, 2011.

FIDEICOMISO DE ESTUDIOS ESTRATEGICOS SOBRE LA CIUDAD DE MEXICO. La ciudad de México, hoy. Ciudad de México: Gobierno del Distrito Federal. Oficialía Mayor, 2002.

GARZA, G. La organización espacial del sector servicios en México. Ciudad de México: El Colegio de México, 2006.

HARVEY, D. The condition of Posmodernity. London: Blackwell, 1989.

Espacios de Esperanza. Madrid: Akal, 2000.

HIERNAUX, D.; TOMAS, F. (Comp.). Cambios económicos y periferia de las grandes ciudades: el caso de la Ciudad de México. México: IFAL-Universidd Autónoma Metropolitana, 1994.

HORNBY, A. Oxford advanced Learner's Dictionary of current English. Oxford: University Press, 1974.

INSTITUTO NACIONAL DE ESTADÍSTICA, GEOGRAFÍA E INFORMÁTICA (INEGI). Anuario estadistico de Comercio Exterior de los Estados Unidos Mexicanos. Aguascalientes: INEGI, 2009.

. Censo Agrícola Ganadero y Forestal. Aguascalientes: INEGI, 2009.

. Anuario estadístico de Comercio Exterior de los Estados Unidos Mexicanos. Aguascalientes: INEGI, 2010.

MACÍAS, J. M. Significado de la vulnerabilidad social frente a los desastres. Revista Mexicana de Sociología, v. 54, n. 5, p. 3-10, oct. 1992. https://doi.org/10.2307/3540934 
MÁRQUEZ, 1. La desindustrialización del área metropolitana de la ciudad de México, 19802004. Tesis (Maestría en Estudios Regionales) - Instituto de Investigaciones Dr. José María Luis Mora, Ciudad de México, 2005.

MERCOSUR. La crisis del campo mexicano, 13 abr. 2011.

MÉXICO. Presidencia de la República. Segundo Informe de Gobierno, Enrique Peńa Nieto. Septiembre, Anexo Estadístico. Ciudad de México. 2014.

PILIEGO, F. Estrategias de desarrollo social en situaciones de desastre. Revista Mexicana de Sociologí, v. 54, n. 4, p. 11-26, oct. 1992. https://doi.org/10.2307/3540935

PRADILLA, E. Los territorios del neoliberalismo en América Latina. México: Miguel Ángel Porrúa; Universidad Autónoma Metropolitana-Xochimilco, 2009.

QUINTANA, 1; EGURROLA, J. I.; NANDAYAPA, C. Encadenamientos productivos, industria y vaciamiento productivo regional en México. In: CALVA, J. 1. (Coord.). Agenda para el desarrollo. México: UNAM; Miguel Ángel Porrua, 2007. p. 267-283.

RAMÍREZ, P.; ZICARDI, A. Pobreza urbana, desigualdad y exclusión en la Ciudad del siglo XXI. Una introducción. In: CORDERA, R.; RAMIREZ, P.; ZICARDI, A. (Coord.). Pobreza, desigualdad y exclusión social en la ciudad del siglo XXI. México: Siglo XXI, 2008. p. 23-48.

RAMÍREZ, B. R. Procesos territoriales, escalas y utopia. Ciudades, v. 15, n. 60, p. 7-14, oct./ dic. 2003.

Del funcionalismo industrial al funcionalismo de servicios: ¿̨la nueva utopía de la metrópoli postindustrial del valle de México? EURE, v. XXXIII, n. 95, p. 61-74, mayo 2006. https://doi.org/10.4067/s0250-71612006000100004

Escalas territoriales y agentes diferenciales en la integración de políticas de desarrollo. In: CALVA, J. 1. (Coord.). Agenda para el desarrollo. México: UNAM; Miguel Ángel Porrua, 2007. p. 123-137.

De la escala al espacio en la construcción del desarrollo regional. In: FERNÁNDEZ, V. R.; BRANDAO, C. (Coord.). Escalas y politicas del desarrollo regional. Desafíos para América Latina. Buenos Aires: Universidad Nacional del Litoral; Miño Dávila, 2010. p. 217-237.

La ciudad gentrificada: del proceso a la adjetivización, ponencia presentada. In: SEMINARIO INTERNACIONAL DE LA RED LATINOAMERICANA DE INVESTIGACIÓN SOBRE TEORÍA URBANA, 3., 2016, Querétaro. Anales... Querétaro: Red Latinoamericana de Investigación sobre Teoría Urbana, 2016 (mimeo).

; PRADILLA, E. El Tratado Norteamericano de Libre Comercio y la integración territorial de México a Estados Unidos. Revista Interamericana de Planificación, v. XXVI, n. 103, p. 34-50, jul./sep. 1993.

REAL ACADEMIA DE LA LENGUA. Diccionario de la Lengua Española. 22. ed. Madrid: Espasa Calpe. 2001.

REVISTAME. Aumentó en un año 35\% uso de suelo para cultivar drogas: Senado, 2011.

RUBIO, B. Explotados y Excluidos. Los campesino latinoamericanos en la fase agroexportadora neoliberal. 2. ed. México: Plaza y Valdés editores, 2003.

SECRETARIA DE AGRICULTURA, GANADERÍA, DESARROLLO RURAL, PESCA Y ALIMENTACIÓN. Cierre de la producción agrícola por estado, Estados Unidos Mexicanos, 2010. México: Secretaria de Agricultura, Ganadería, Desarrollo Rural, Pesca y Alimentación, 2010.

SINGER, P. Economía politica de la urbanización. México: Siglo XXI, 1975. 